\title{
Effect of Septoplasty on Objective and Subjective Parameters of Osas Treatment
}

\author{
Ela Araz Server ${ }^{1}$ (D) Engin Acıoğlu² (D), Özgür Yiğit ${ }^{3}$ (D) Ecem Sevim Akı $^{4}$ (D) Nihal Seden $^{3}$ (D), \\ Ahmet Görkem Yasak $^{5}$ (D)
}

\begin{abstract}
${ }^{1}$ Istinye University, Department of Otorhinolaryngology/Head and Neck Surgery, Istanbul, Turkey ${ }^{2}$ Memorial Atasehir Hospital, Department of Otorhinolaryngology/Head and Neck Surgery, Istanbul, Turkey ${ }^{3}$ Istanbul Training and Research Hospital, Department of Otorhinolaryngology/Head and Neck Surgery, Istanbul, Turkey ${ }^{4}$ Bozyaka Training and Research Hospital, Department of Otorhinolaryngology/Head and Neck Surgery, Izmir, Turkey ${ }^{6}$ Kilis State Hospital, Department of Otorhinolaryngology/Head and Neck Surgery, Kilis, Turkey
\end{abstract}

ORCID ID: E.A.S. 0000-0002-8462-3605; E.A. 0000-0002-1619-7851; Ö.Y. 0000-0003-1731-3233; E.S.A. 0000-0001-6256-2015; N.S. 0000-0003-0137-1535; A.G.Y. 0000-0003-1207-5846

Citation: Araz Server E, Acioglu E, Yigit O, Aki ES, Seden N, Yasak AG. Effect of septoplasty on objective and subjective parameters of osas treatment. Tr-ENT 2021;31(1):10-5. https://doi.org/10.26650/Tr-ENT.2021.41275

\begin{abstract}
Objective: The aim of this study was to investigate the effect of isolated septoplasty on polysomnography (PSG) data and subjective findings in patients with OSAS.

Materials and Methods: A total of 25 patients with symptoms of nasal obstruction, septum deviation and who had been diagnosed with OSAS, were included in the study. All patients underwent isolated septoplasty. The PSG prior to and 6 months after the operation, were recorded. The Epworth sleepiness scale (ESS), the Pittsburgh Sleep Quality Index (PSOI) and Snore Outcomes Survey were performed prior to and 6 months after the operation.

Results: The postoperative apnea-hypopnea index (AHI) score, minimum oxygen saturation (MOS) and the snoring time were statistically significantly decreased compared to the preoperative measurements $(p=0,000, p=0,01$ and $p=0,000$, respectively). The rate of patients with a decrease in the $\mathrm{AHI}$ index of more than $50 \%$, was $56 \%$. A significant decrease was observed in the ESS and snore outcome survey measurements compared to the preoperative measurements. In PSQI, all sub-factor scores except postoperative medication were determined to be significantly decreased compared to the preoperative period $(p<0.05)$. No correlation was observed between AHI and ESS, snore outcome survey and the PSQI reduction ( $p>0.05)$.

Conclusion: Isolated septoplasty leads to an improvement in the subjective and objective parameters of the patients with OSAS and symptoms of nasal obstruction, although not to a complete recovery.
\end{abstract}

Keywords: Obstructive sleep apnea syndrome, snoring, polysomnography, apnea-hypopnea index, septoplasty

\section{INTRODUCTION}

Obstructive Sleep Apnea Syndrome (OSAS) is characterized by partial or complete repetitive obstructions of the upper respiratory tract (URT) during sleep (1). The most common symptoms of OSAS include snoring, witnessed apnea, feeling of excessive sleepiness during daytime, and waking up with a feeling of asphyxia and insomnia (2). Snoring is the most common cause of patient admission. Patients with OSAS almost always complain of snoring (a minimum of 5 nights a week or more), and the tract is blocked due to frequent apneas (3). Although the pathogenesis of OSAS or snoring has not been completely understood, basic anatomic properties related to obstruction of the URT have been determined (4). The obstruction in the URT may be at any level from the nasal cavity to the larynx. Obstructions of the nasal cavity occupy an important extent of this mechanism (5).

The nose is responsible for approximately $70 \%$ of URT resistance in the adult, and for the majority of URT resistance

Corresponding Author: Ecem Sevim Akı E-mail: ecem.longur@gmail.com

Submitted: 03.12.2020 • Revision Requested: 24.02.2021 • Last Revision Received: 23.05.2021 • Accepted: 30.05.2021

This work is licensed under Creative Commons Attribution-NonCommercial 4.0 International License. 
during awakeness (6). It is known that nasal obstruction related to mechanical (such as septal deviation or nasal polyps) or inflammatory/vasomotor (such as acute or chronic rhinitis) causes contribute to OSAS and snoring. Many studies have demonstrated the importance of correction of these pathologies via medical or surgical methods in the treatment of OSAS and snoring. Among these studies, some have reported no improvement in the objective parameters of OSAS measured by Polysomnography (PSG) despite the improvements in subjective parameters such as the Epworth $S$ leepiness $S$ cale (ESS), snoring and quality of life; on the other hand, some studies have reported improvement in the objective parameters as well (7-9). In most of these studies, nasal surgeries were studied. However, there are many different pathologies which lead to nasal obstruction and many surgical methods to treat these pathologies, such as septoplasty, septorhinoplasty, turbinoplasty, valve surgery, functional sinus surgery.

Nasal septum deviation is the most frequent pathology of nasal obstruction and the most frequent surgery performed for this pathology is septoplasty. The number of studies investigating the effect of isolated septoplasty on the PSG measurements and subjective findings in patients with OSAS is limited. In our study, we aimed to investigate the effect of isolated septoplasty on snoring, quality of sleep and PSG measure parameters in patients with OSAS and nasal obstruction, and to assess whether the changes observed in subjective parameters were correlated with those observed in PSG measurements or not.

\section{MATERIALS AND METHODS}

Ethical approval was obtained from the local committee. Patients included in the study were informed and written informed consents were obtained from each. Patient selection was made upon investigation in the sleep laboratory. All patients underwent a complete ear-nose-throat examination. The patients were asked to answer the question whether they had nasal obstruction or not. Those who answered this question as 'yes', those who were found to have nasal septum deviation and those with an apnea-hypo pnea index (AHI) of $>5$ were included in the study if they were between 18 and 60 years of age.
Those with no nasal obstruction, septum deviation, those who had additional nasal pathologies such as sinusitis, nasal polyp, concha hypertrophy or nasal valve failure, those who required an additional surgical procedure other than septoplasty and those with a systemic disease were excluded from the study. Patients with malocclusion, retrognathia, tongue base hypertrophy, grade 2 tonsil hypertrophy, laryngeal pathology, and obstruction sites identified in Muller's maneuver were also excluded from the study.

The patients were classified according to the septal deviation types described by Baumann and Baumann (10) (Figure 1). Types of septal deviation, body mass index (BMI) and demographic characteristics of the patients were recorded. Then they were classified as mild, moderate and severe OSAS according to their AHI indexes. All patients underwent septoplasty under general anesthesia.

The patients underwent an ear-nose-throat examination 6 months after the operation. Their complaints of nasal obstruction were questioned. Patients who experienced complications such as septum perforation, septo conchal synechiae or re-deviation were excluded from the study. The PSGs of the patients were repeated. Their BMIs, AHI in PSGs, minimum oxygen saturation values (MOS) and snoring counts were recorded, both prior to and 6 months after the operation. The ESS, Pittsburgh Sleep Quality Index (PSQI), and the snoring outcome survey were fulfilled. All measurements were compared for pre- and post-operative periods.

\section{Statistical method}

The $\mathrm{G}^{*}$ power program was used for power analysis. Descriptive statistics were analyzed using mean, standard deviation, median, minimum and maximum values, frequency and ratios. Distribution of the variables were analyzed using the Kolmogorov Simirnov test. The Mann-Whitney $U$ test was used for analysis of the independent quantitative data. The Paired samples $t$ test and the Wilcoxon tests were used for analysis of the dependent quantitative data. The MC Mac Nemar test was used for the analysis of dependent qualitative data. The analyses were performed using the SPSS 22.0 program package.
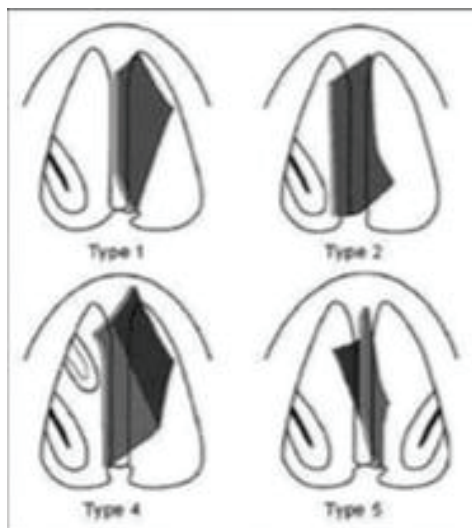

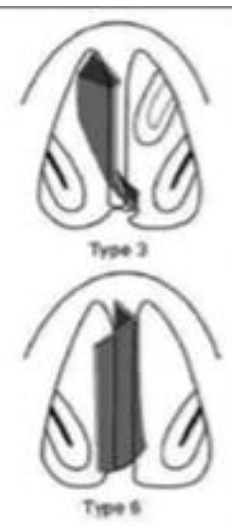

Figure 1: C lassification of the septal deviation types according described by Baumann and Baumann (10) 


\section{RESULTS}

A total of 25 patients between 24 and 62 years (mean: 45) of age were included in the study. Among these, 17 were male and 8 were female. The demographic characteristics and distribution of the patients according to the OSAS degree and the region of septal deviation are summarized in Table 1. The complaint of nasal obstruction had disappeared in all patients in the postoperative $6^{\text {th }}$ month, and rhinoscopic examinations revealed that the septum were in the midplane. No complications such as perforation or synechia were observed. Thus, no patient was excluded.

In the power analysis based on other sample studies, the power of our study was found to be 0.96 (effect size:0.80, $\alpha$ error:0.05). No significant change was observed in the BMI measurements obtained preoperatively compared to those obtained postoperatively $(p=0.16)$. The PSG values revealed a decrease in postoperative $\mathrm{AHI}$ scores, MOS and snoring time compared to the preoperative values $(p=0,000, p=0,01$ and $p=0,000$, respectively). Among the 5 patients with mild OSAS included in the study, 1 experienced complete improvement. Seven of the 9 patients with moderate OSAS turned into mild OSAS, 4 of 11 patients with severe OSAS turned into moderate OSAS, and 3 of 11 patients turned into mild OSAS postoperatively. A total of 14 patients demonstrated a decrease of more than $50 \%$ in the $\mathrm{AHI}$ index, which constituted $56 \%$ of all patients.

A significant decrease was observed in the ESS and the snoring outcome survey postoperatively compared to the preoperative
Table 1: Demographic characteristics, septum deviation classifications and distribution of OSAS classifications of the patients included in the study.

\begin{tabular}{|c|c|c|c|c|c|}
\hline \multirow[b]{2}{*}{ Age } & & \multirow{2}{*}{$\begin{array}{l}\text { Min-Max } \\
24-62\end{array}$} & \multirow{2}{*}{$\begin{array}{l}\text { Median } \\
45\end{array}$} & \multicolumn{2}{|c|}{ Average $\pm S D / n-\%$} \\
\hline & & & & 43.3 & \\
\hline \multirow{2}{*}{ Gender } & Male & & & 17 & $68.0 \%$ \\
\hline & Female & & & 8 & $32.0 \%$ \\
\hline \multirow{6}{*}{$\begin{array}{l}\text { Deviation } \\
\text { Region }\end{array}$} & 1 & & & 4 & $16.0 \%$ \\
\hline & 2 & & & 7 & $28.0 \%$ \\
\hline & 3 & & & 3 & $12.0 \%$ \\
\hline & 4 & & & 5 & $20.0 \%$ \\
\hline & 5 & & & 1 & $4.0 \%$ \\
\hline & 6 & & & 5 & $20.0 \%$ \\
\hline \multirow{3}{*}{ OSAS } & Mild & & & 5 & $20.0 \%$ \\
\hline & Moderate & & & 9 & $36.0 \%$ \\
\hline & Severe & & & 11 & $44.0 \%$ \\
\hline
\end{tabular}

measurements $(p=0.000$ and $p=0.000$, respectively). The postoperative PSQI score significantly decreased for the subjective sleep quality, sleep latency, duration of sleep, efficacy of sleep, sleeping disorder and the daytime functions subfactor scores compared to the preoperative period $(p<0.05)$, whereas the medication was not changed significantly compared to the preoperative period $(p=1.00)$. Comparisons between the preoperative measurements and those obtained at the $6^{\text {th }}$ month are summarized in Table 2.

Table 2: Comparison of pre- and post-operative PSG outcomes, ESS, snoring outcome survey and PSQI.

\begin{tabular}{|c|c|c|c|c|c|}
\hline & \multicolumn{2}{|l|}{ Preop } & \multicolumn{2}{|c|}{ Postop } & \multirow{2}{*}{ p } \\
\hline & Average. $\pm S D / n-\%$ & Median & Average $\pm S D / n-\%$ & Median & \\
\hline BMI & $27.6 \pm 4.9$ & 28.3 & $27.4 \pm 4.6$ & 28.2 & $0.156^{t}$ \\
\hline \multicolumn{6}{|l|}{ OSAS } \\
\hline Snoring & 0.0 & & $16.0 \%$ & & $0.000^{\mathrm{N}}$ \\
\hline Mild & $20.0 \%$ & & $48.0 \%$ & & \\
\hline Moderate & $36.0 \%$ & & $24.0 \%$ & & \\
\hline Severe & $44.0 \%$ & & $12.0 \%$ & & \\
\hline $\mathrm{AHI}$ & $29.2 \pm 17.5$ & 23.0 & $15.6 \pm 11.7$ & 10.5 & $0.000^{\mathrm{w}}$ \\
\hline MOS & $80.0 \pm 10.9$ & 83.0 & $84.4 \pm 9.9$ & 86.0 & $0.010^{\mathrm{w}}$ \\
\hline Snoring Count & $183.4 \pm 117.5$ & 180.0 & $81.8 \pm 77.3$ & 65.7 & $0.000^{\mathrm{w}}$ \\
\hline ESS Total & $10.8 \pm 5.8$ & 12.0 & $6.6 \pm 4.1$ & 6.0 & $0.000^{\mathrm{w}}$ \\
\hline Snoring Outcome Survey & $29.6 \pm 3.7$ & 30.0 & $20.8 \pm 3.5$ & 22.0 & $0.000^{\mathrm{w}}$ \\
\hline Subjective Sleep Quality & $2.2 \pm 0.5$ & 2.0 & $1.2 \pm 0.4$ & 1.0 & $0.000^{\mathrm{w}}$ \\
\hline Sleep Latency & $2.1 \pm 1.6$ & 2.0 & $1.1 \pm 0.9$ & 1.0 & $0.002^{\mathrm{w}}$ \\
\hline Sleep Duration & $0.8 \pm 0.8$ & 1.0 & $0.4 \pm 0.5$ & 0.0 & $0.013^{w}$ \\
\hline Habitual Sleep Efficiency & $0.7 \pm 0.7$ & 1.0 & $0.2 \pm 0.4$ & 0.0 & $0.001^{w}$ \\
\hline Sleep Disturbances & $1.8 \pm 0.6$ & 2.0 & $1.0 \pm 0.4$ & 1.0 & $0.000^{\mathrm{w}}$ \\
\hline Use of Sleeping Medication & $0.3 \pm 0.7$ & 0.0 & $0.3 \pm 0.8$ & 0.0 & $1.000^{\mathrm{w}}$ \\
\hline Daytime Dysfunction & $2.3 \pm 1.1$ & 2.0 & $1.1 \pm 0.8$ & 1.0 & $0.000^{\mathrm{w}}$ \\
\hline Total Score & $10.5 \pm 2.9$ & 11.0 & $5.3 \pm 1.7$ & 5.0 & $0.000^{\mathrm{w}}$ \\
\hline
\end{tabular}

'The Paired samples t test, wWilcoxon test, ${ }^{\mathrm{N}}$ McNemar test. AHI: apnea-hypopnea index, MOS: the minimum oxygen saturation, ESS: Epworth sleepiness scale, PSQI: Pittsburgh Sleep Quality Index, PSG: Polysomnography, BMI: Body mass index 
A significant positive correlation was observed between the decrease in $\mathrm{AHI}$ and the decrease in the snoring count according to PSG postoperatively $(p<0.05)$. However, no correlation was observed between the reductions in $\mathrm{AHI}$ and MOS. No correlation was determined between AHI and ESS, snoring outcome survey, total PSQI score, subjective quality of index, sleep latency, duration of sleep, efficacy of sleep, sleeping disorder, daytime functions and the decrease in the snoring outcome survey scores ( $p>0.05$ ) (Table 3 ).

Table 3: Correlation of changes in pre- and post-operative subjective parameters with those in $\mathrm{AHI}$.

\begin{tabular}{|c|c|c|}
\hline & & AHI Changes \\
\hline \multirow{2}{*}{ Epworth Total } & $r$ & 0.374 \\
\hline & $\mathrm{p}$ & 0.065 \\
\hline \multirow{2}{*}{ Subjective Sleep Quality } & $r$ & 0.085 \\
\hline & $\mathrm{p}$ & 0.688 \\
\hline \multirow{2}{*}{ Sleep Latency } & $r$ & 0.256 \\
\hline & $\mathrm{p}$ & 0,216 \\
\hline \multirow{2}{*}{ Sleep Duration } & $r$ & 0.258 \\
\hline & $\mathrm{p}$ & 0,213 \\
\hline \multirow{2}{*}{ Habitual Sleep Efficiency } & $r$ & 0.087 \\
\hline & $\mathrm{p}$ & 0.680 \\
\hline \multirow{2}{*}{ Sleep Disturbances } & $r$ & 0.112 \\
\hline & $\mathrm{p}$ & 0.593 \\
\hline \multirow{2}{*}{ Use of sleeping medication } & $r$ & 0.228 \\
\hline & $\mathrm{p}$ & 0.272 \\
\hline \multirow[b]{2}{*}{ Daytime Dysfunction } & $r$ & -0.057 \\
\hline & $\mathrm{p}$ & 0.787 \\
\hline \multirow{2}{*}{ Total Score } & $r$ & 0.388 \\
\hline & $\mathrm{p}$ & 0.056 \\
\hline \multirow{2}{*}{ Snoring Outcome Survey } & $r$ & 0.274 \\
\hline & $\mathrm{p}$ & 0.185 \\
\hline
\end{tabular}

\section{DISCUSSION}

In our study, which aimed to investigate the effect of isolated septoplasty on the PSG outcomes, snoring and quality of sleep in patients with OSAS, nasal obstruction and septal deviation, a significant improvement was observed in the $\mathrm{AHI}, \mathrm{MOS}$, snoring time, ESS, snoring outcome survey and the PSQI scores following isolated septoplasty. However, no significant correlation was observed between the change in $\mathrm{AHI}$ and quality of sleep and the snoring measures. This suggests that objective and subjective data should be independently evaluated in patients with OSAS.

Independent from the severity of OSAS, first-line therapy is a general precaution (such as I osing weight, position of sleep, avoiding alcohol and sedatives or treatment of concomitant diseases), whereas Continuous Positive Airway Pressure (CPAP) therapy, intraoral devices and surgical treatment are the other treatment options (11). Nasal surgeries constitute an important part of surgical treatments. The aim of OSAS therapy is to improve the quality of sleep and the related quality of life, and most importantly, to contribute to survival by preventing the morbidity caused by OSAS. The efficacy of the treatment is investigated subjectively by using surveys on the quality of sleep and life, and objectively by using the parameters of PSG such as AHI, Respiratory Disturbance Index (RDI) or MOS (11). The effect of nasal surgeries on the treatment of OSAS has been investigated using these parameters in different studies. However, conflicting outcomes have been observed in these studies. Thus, the effect of nasal surgeries on the treatment of OSAS is yet controversial.

The meta-analysis of Ischi et al. revealed improvement in ESS and RDI following nasal surgery, whereas no change was observed in $\mathrm{AHI}$ (7). On the contrary, the meta-analysis of Jun Wu et al. revealed significant improvement in both $\mathrm{AHI}$ and ESS following isolated nasal surgery, where the improvement in $\mathrm{AHI}$ was less (9). Although isolated nasal surgery is the subject in the studies evaluated in these meta-analyses, it has been emphasized that the surgical methods vary to an important extent and that heterogeneity is significant. Meen et al. discussed the heterogeneities in nasal surgeries, and stated that surgical treatments were not standardized between studies or individually, and that patients underwent various types of surgeries and combinations of surgeries (12). In conclusion, different from the meta-analysis of Jun Wu et al., they emphasized that nasal surgeries are ineffective in improving $\mathrm{AHI}$ and other objective sleep parameters of OSAS safely, and that improvement was observed in subjective parameters such as snoring (5). In most other studies and metaanalyse s, improvement in AHI after nasal surgery was minimal or not significant. However, in this study, mean AHI changed from 29.2 to 15.6 , nearly $50 \%$. In our opinion, for significant improvement in $\mathrm{AHI}$ after isolated septoplasty, patients should have high nasal resistance and minimal (or no) retro-pharyngeal or retroglossal obstruction. One of the limitations of our study wa s not using objective nasal obstruction.

Although nasal surgeries are numerous in number, studies have mostly included patients undergoing septoplasty and septoplasty combined with turbinoplasty or turbinectomy. Park et al. performed septoplasty and turbinoplasty on 25 patients with OSAS, and reported that the operations led to improvements in the nasal airway clearance postoperatively, and reduced the severity of the disease in $56 \%$ of the patients (13). In the study of Moxness et al.comparing septoplasty and turbinoplasty, 33 of 59 patients underwent septoplasty only, whereas 26 underwent septoplasty and inferior concha volumereducing surgeries (14). AHI was observed to have reduced in the group undergoing septoplasty and turbinoplasty, whereas no change was observed in $\mathrm{AHI}$ in the group undergoing septoplasty only. In our study, we aimed to homogenize our group by including patients undergoing septoplasty only. In contrast to the study of Moxness et al., we observed a reduction in $\mathrm{AHI}$ of more than $50 \%$ in $56 \%$ of the patients. 
In the literature, success in surgical treatment of OSAS is generally accepted as a reduction in the $\mathrm{AHI}$ of more than $50 \%$ or a final $\mathrm{AHI}$ of less than $20 / \mathrm{h}$. However, a reduction in $\mathrm{AHI}$ would lead to a decrease in the mortality and morbidity of the disease and an increase in the quality of life of the patient (11). Therefore, the success of nasal surgeries should be evaluated not only by the PSG findings, but improvement in CPAP use, psychological situations such as depression, daytime sleepiness, quality of sleep and snoring should be taken into account as well.

Although conflicting outcomes of PSG have been reported in studies demonstrating the effect of nasal surgeries on OSAS, many studies are in agreement for its positive effects on ESS, snoring and quality of life (15-17). ESS is the most frequently used subjective survey in patients with OSAS. Improvement has been observed in ESS following nasal surgeries in most of the conducted studies (7-9). In our study, too, a significant improvement was observed in ESS. Similar to our study, Lorente et al. showed recovery of AHI and ESS after septoplasty in 34 OSAS patients (18). However, in our study, no correlation was observed between this improvement in ESS and the improvement in AHI.

One of the most contentious subjective findings is snoring. Bury et al. emphasized that nasal surgeries may not provide a precise solution for snoring, although they may lead to an improvement, and that palatal pathologies, which play an important role in the pathogenesis of snoring, should be considered as well (15). In the study of Wu et al. investigating the effect of nasal surgeries on subjective findings, a significant reduction was observed in the visual analog scale of nasal obstruction, ESS, snoring outcome survey, bedroom partner survey and the Sino-Nasal Outcome Test scores (16). Likewise, it was observed in the study of Kalaycik et al. that Nasal Obstruction Symptom Evaluation scale (NOSE), ESS, and Snore Symptom Inventory (SSI) scores improved in patients with habitual snoring and nasal obstruction following septoplasty (17). However, no correlation was observed between score and the degree of septum deviation . In our study, we used snoring time and the snoring outcome survey in PSG to evaluate the effect of septoplasty both objectively and subjectively. We demonstrated an objective and subjective improvement following septoplasty. However, while we did not observe any patients with a complete recovery, as emphasized in the review of Bury et al., we did observe an improvement in snoring.

Quality of sleep is an important parameter for patients with OSAS, which impairs the quality of life. The "Pittsburgh Sleep Quality Index (PSQI)", which is a reliable and consistent survey widely used in determining the quality of sleep within the last 1 month, has been used in many patient populations (19). In the study of Stapleton et al., demonstrating the effect of nasal surgery on the quality of sleep, it was found that PSQI better correlated with the Sleep Quality Likert Scores than to ESS (20). They reported that a secondary improvement would be observed in the quality of sleep following nasal surgeries, and that the degree of improvement in the quality of sleep would correlate with the severity of preoperative nasal obstruction and the degree of improvement in the obstruction by surgery. In our study, we observed a significant improvement in the sleep quality of patients with symptoms of nasal obstruction following septoplasty as well.

In studies evaluating subjective parameters, surveys grading nasal obstruction such as NOSE or the Sino-Nasal Outcome Index were compared to the subjective parameters of OSAS, and significant correlations were determined $(16,17,19)$. In our study, we aimed to investigate the correlation between improvements in the objective and subjective parameters of OSAS, and thus, we did not plan an additional survey for nasal obstruction. One of the limitations of our study is not using these surveys evaluating nasal obstruction. The other limitations include small sample size, impossibility of statistical evaluation of the septal deviation site due to the insufficient number of cases, and lack of comparison for evaluation of the deviation and obstruction findings by degrees.

Our study is important since it provides a homogeneous distribution of patients undergoing isolated septoplasty, due to nasal obstruction symptoms. Furthermore, our study is the first to evaluate OSAS with both objective and subjective parameters, and to demonstrate that the improvement in subjective parameters is independent from $\mathrm{AHI}$.

\section{CONCLUSION}

Our study demonstrated that isolated septoplasty provided positive effects on PSG findings such as AHI, MOS or snoring time, and ESS, PSQI and the snoring outcome survey in patients with OSAS describing nasal obstruction and diagnosed to have septal deviation. However, the improvements in subjective parameters do not correlate to the improvements in $\mathrm{AHI}$. Therefore, objective and subjective parameters should be independently evaluated for the efficacy of septoplasty in patients with OSAS.

Ethics Committee Approval: Ethical approval was obtained from the Istanbul Training and Research Hospital Clinical Research Ethics Committee (2011-KAEK-50).

Peer Review: Externally peer-reviewed.

Informed Consent: Patients included in the study were informed and written informed consents were obtained from each.

Author Contributions: Conception/Design of Study- E.A.S., E.A., Ö.Y., E.S.A., N.S., A.G.Y.; Data Acquisition- E.A.S., E.A., Ö.Y., E.S.A., N.S., A.G.Y.; Data Analysis/Interpretation- E.A.S., E.A., Ö.Y., E.S.A., N.S., A.G.Y.; Drafting Manuscript- E.A.S., E.A., Ö.Y., E.S.A., N.S., A.G.Y.; Critical Revision of Manuscript- E.A.S., E.A., Ö.Y., E.S.A., N.S., A.G.Y.; Final Approval and Accountability- E.A.S., E.A., Ö.Y., E.S.A., N.S., A.G.Y.

Conflict of Interest: Authors declared no conflict of interest.

Financial Disclosure: Authors declared no financial support. 


\section{REFERENCES}

1. Gaudette E, Kimoff RJ. Pathophysiology of OSA. Eur Respir Mon 2010;50:31-50.

2. Malow BA. Approach to the patient with disordered sleep In: Kryger MH, Roth T, Dement WC, eds. Principles and Practice of Sleep Medicine. 4th Ed. Philadelphia, WB Saunders 2005;589-93.

3. Schlosshan D, Elliott MW. Clinical presentation and diagnosis of the obstructive sleep apnea hypopnea syndrome. Thorax 2004;59:34752.

4. Schwab RJ, Remmers JE, Kuna ST. Chapter 101 - Anatomy And Physiology of Upper Airway Obstruction. In: Kryger MH, Roth T, Dement WC (eds). Principles and Practice of Sleep Medicine, 5th ed. Missouri: Elsevier Saunders 2011:1153-71.

5. Gharibeh T, Mehra R. Obstructive sleep apnea syndrome: natural history, diagnosis, and emerging treatment options. Nat Sci Sleep 2010;2:233-55.

6. Woodson BT. Sleep Medicine and Surgery. In: James Snow, P. Ashley Wackym, ed. Ballenger's Otolaryngology, Head and Neck Surgery, centennial ed. New York : People's Medical Publishing House. BC Decker Inc. 2009:983-96.

7. Ishii L, Roxbury C, Godoy A, et al. Does nasal surgery improve OSA in patients with nasal obstruction and OSA? A meta-analysis. Otolaryngol Head Neck Surg 2015;153:326-33.

8. Li HY, Wang PC, Chen YP, et al. Critical appraisal and meta-analysis of nasal surgery for obstructive sleep apnea. Am J Rhinol Allergy 2011;25:45-9.

9. Wu J, Zhao G, Li Y, Zang H, Wang T, Wang D, Han D. Apneahypopnea index decreased significantly after nasal surgery for obstructive sleep apnea: A meta-analysis. Medicine (Baltimore). 2017;96(5):e6008

10. Baumann I, Baumann H. A new classification of septal deviations. Rhinology 2007;45:220-3.
11. Mickelson SA. Nasal Surgery for Obstructive Sleep Apnea Syndrome. Otolaryngol Clin North Am 2016;49(6):1373-81.

12. Meen EK, Chandra RK. The role of the nose in sleep disordered breathing. Am J Rhinol Allergy 2013;27(3):213-20.

13. Park $\mathrm{CY}$, Hong JH, Lee JH, et al. Clinical effect of surgical correction for nasal pathology on the treatment of obstructive sleep apnea syndrome. PloSOne 2014;9:e9876.

14. Moxness $\mathrm{MH}$, Nordgård $\mathrm{S}$. An observational cohort study of the effects of septoplasty with or without inferior turbinate reduction in patients with obstructive sleep apnea. BMC Ear Nose Throat Disord 2014;14:11.

15. Bury SB, Singh $A$. The role of nasal treatments in snoring obstructive sleep apnoea . Curr Opin Otolaryngol Head Neck Surg 2015;23:39-46.

16. Wu J, Zang HR, Wang T, Zhou B, Ye JY, Li YC, Han DM. Evaluation of the subjective efficacy of nasal surgery. J Laryngol Otol 2017;131(1):37-43.

17. Ertugay CK, Toros SZ, Karaca CT, Kulekci S, Verim A, Ertugay OC, Naiboglu B. Is septoplasty effective on habitual snoring in patients with nasal obstruction ?Eur Arch Otorhinolaryngol. 2015;272(7):1687-91.

18. Lorente J, Jurado MJ, Romero O, Quesada P, Quesada JL, Sagalés T. Effects Of Functional Septoplasty in obstructive sleep apnea syndrome. Med Clin (Barc). 2005;125(8):290-2.

19. Buysse DJ, Hall ML, Strollo PJ, et al. Relationships between the Pittsburgh Sleep Quality Index (PSQI), Epworth Sleepiness Scale (ESS), and clinical/polysomnographic measures in a community sample. J Clin Sleep Med 2008;4:563-71.

20. Stapleton AL, Chang YF, Soose RJ, Gillman GS. The impact of nasal surgery on sleep quality: a prospective outcomes study. Otolaryngol Head Neck Surg 2014;151(5):868-73. 